\title{
Mapping of genetic loci predisposing to hypertriglyceridaemia in the hereditary hypertriglyceridaemic rat: analysis of genetic association with related traits of the insulin resistance syndrome
}

\author{
I. Klimes ${ }^{1}$, K. Weston ${ }^{2}$, P. Kovacs ${ }^{3}$, D. Gasperikova ${ }^{1}$, D. Jezova ${ }^{1}$, R. Kvetnansky ${ }^{1}$, J. R. Thompson ${ }^{4}$, \\ E. Sebokova1, N. J. Samani \\ ${ }^{1}$ Diabetes and Nutrition Research Laboratory, Institute of Experimental Endocrinology, Slovak Academy of Sciences, Bratislava, \\ Slovakia \\ 2 Division of Cardiology, Department of Medicine, University of Leicester, UK \\ ${ }^{3}$ Phoenix Epidemiology and Clinical Research Branch, NIDDK, NIH, Phoenix, AZ, USA \\ ${ }^{4}$ Department of Epidemiology and Public Health, University of Leicester, UK
}

\section{Abstract}

Aims/hypothesis. Hypertriglyceridaemia is an important risk factor for coronary heart disease, especially in the context of the insulin resistance syndrome where it often occurs with hypertension. The two phenotypes are also associated in the hereditary hypertriglyceridaemic (hHTg) rat. The aim of this study was to map quantitative trait loci that affect plasma triglyceride concentration in the hHTg rat and determine whether they co-localize with loci for blood pressure. Methods. Second filial generation progeny $(n=189)$ from a cross of the hHTg rat with the Brown Norway rat were phenotyped for fasting plasma triglyceride, glucose and insulin concentrations, and direct unrestrained resting blood pressure. A partial genome-scan was conducted using 153 microsatellite markers that were polymorphic between the two strains.

Results. A major locus (lod score 6.5) influencing plasma triglyceride concentration in a co-dominant fashion was mapped to chromosome 4 between D1Mit5 and D1Mit17. Chromosome 8 contained multiple peaks with a lod score greater than 4.0 influencing triglyceride concentration. Importantly, none of the triglyceride loci had an effect on blood pressure. The triglyceride locus on chromosome 4 co-localized with a locus for fasting plasma insulin (lod score 4.1), although the effect on insulin concentration was in the opposite direction to that on triglyceride.

Conclusion/interpretation. We have mapped the major loci that affect plasma triglyceride concentration in the hHTg rat. These loci do not influence blood pressure suggesting that these commonly associated phenotypes of the insulin resistance syndrome are not be due to pleiotropic effects of the same gene(s). [Diabetologia (2003) 46:352-358]

Keywords Hypertriglyceridaemia, insulin resistance syndrome, hypertension, quantitative trait loci, genetic linkage.
In the last few years the insulin resistance (IR) syndrome has emerged as a potent risk factor for coronary heart disease $[1,2]$.The precise association and the possible causal relationships between the major traits

Received: 16 July 2002 / Revised: 11 October 2002

Published online: 1 March 2003

C Springer-Verlag 2003

Corresponding author: Dr. N. J. Samani, Division of Cardiology, University of Leicester, Clinical Sciences Wing, Glenfield Hospital, Groby Road, Leicester, LE3 9QP UK

E-mail: njs@le.ac.uk

Abbreviations: hHTg rat, hereditary hypertriglyceridemic rat; $\mathrm{F}_{2}$, second filial generation; QTL, quantitative trait locus; $\mathrm{Tg}$, triglyceride. of the syndrome - hypertriglyceridaemia, hyperinsulinaemia, insulin resistance, glucose intolerance, hypertension and other metabolic disturbances such as hyperuricaemia - remain to be clarified [2]. There is substantial evidence that individual traits of the IR syndrome have important genetic determinants and indeed data that the syndrome itself has a major genetic contribution [2,3] Therefore, one possible explanation for the association of the traits of the IR syndrome is that they represent pleiotropic or sequential effects of the same gene variants.

The hereditary hypertriglyceridaemic ( $\mathrm{hHTg}$ ) rat manifests several features of the insulin resistance syndrome except obesity [4]. The strain was developed from Wistar rats on the basis of an ability to at- 
tain a marked rise in plasma triglyceride ( $\mathrm{Tg}$ ) concentration by increased intake of monosaccharides in the diet [5] although the inbred hHTg strain shows increased $\mathrm{Tg}$ concentration compared with control strains on normal rat chow [4]. Further characterisation of the hHTg strain showed that, in addition to being hypertriglyceridaemic it has insulin resistance, hypertension and mild hyperuricaemia [6, 7]. Although the hHTg strain has been the subject of extensive physiologic and biochemical investigation [4], little is known about the primary genetic lesions responsible for the hypertriglyceridaemia and the other features of the insulin resistance syndrome manifested by the strain. Thus, the main aim of this study was to map the major quantitative trait loci (QTLs) affecting $\mathrm{Tg}$ concentration in the hHTg rat and to explore the genetic relationships between plasma $\mathrm{Tg}$, glucose and insulin concentrations s and blood pressure. Co-segregation analysis was carried out on a cohort of $\mathrm{F}_{2}$ hybrids bred from the hHTg (disease-prone) and Brown Norway (disease-resistant) rats.

\section{Materials and Methods}

Rat strains. The derivation of the hHTg strain is described elsewhere [4]. The rats used in this study were from a colony set up at the Institute of Experimental Endocrinology in Bratislava, Slovakia in 1993 from breeding pairs provided by Dr. Vrana, and subsequently maintained by sibling mating. At the time of the initiation of the current study, the animals were in the $35^{\text {th }}$ generation of strict brother-sister mating. Brown Norway (BN) rats were purchased from Charles River Laboratories (Lyon, France). Male and female hHTg rats were reciprocally crossed with the $\mathrm{BN}$ rats to produce (hHTgxBN) and (BN x hHTg) $\mathrm{F}_{1}$ hybrids, which were further inter-crossed to generate $F_{2}$ hybrid populations. Only male $F_{2}$ rats were studied. No phenotypic differences were found between the two reciprocal $F_{2}$ crosses for the traits studied. Altogether, 189 $\mathrm{F}_{2}$ hybrids were analysed. All parental and hybrid animals were kept in the same animal unit under the same environmental conditions. They had free access to tap water and standard laboratory chow and were maintained on a 12-h light and dark cycle $(6 \mathrm{am} / 6 \mathrm{pm})$.

Phenotyping. All procedures followed the principles of laboratory animal care (NIH publication No. 86-23, revised 1985) and the guidelines for the care and use of laboratory animals of the Institute of Experimental Endocrinology, Slovak Academy of Sciences, Bratislava, Slovakia. Phenotyping was carried out at 4 months of age. Glucose, insulin and $\mathrm{Tg}$ concentrations were measured in plasma obtained from the tail vein blood after an overnight starvation. Next day an indwelling catheter was placed into the carotid artery for blood pressure measurement. Briefly, under anaesthesia (ketamine hydrochloride, $70 \mathrm{mg} / \mathrm{kg}$ and xylazine hydrochloride, $10 \mathrm{mg} / \mathrm{kg}$ ), a sterile silastic cannula $0.51 \mathrm{~mm}$ internal diameter (Dow Corning, Midland, Mich., USA) filled with heparin $10 \mathrm{IU} / \mathrm{ml}$ in $0.9 \%$ $\mathrm{NaCl}$ was placed into the right carotid artery, exteriorised through an incision in the neck, closed with a pin and fixed to skin by a suture. Animals were allowed to recover from surgery for at least 2 days. Systolic and diastolic pressure values were then obtained from conscious unrestrained rats using a computer-assisted system, (DBP 1001, Kent Scientific, Lichtfield, Conn., USA). Mean blood pressure values were calculated from 30 measurements made over a 30 min period, starting $1 \mathrm{~h}$ after connection of the carotid catheter to the system. In addition to the $\mathrm{F}_{2}$ rats, $10 \mathrm{hHTg}$ and $10 \mathrm{BN}$ rats were also studied to confirm differences between the parental strains.

Biochemical analyses. Plasma Tg, glucose and insulin concentrations were measured using commercially available kits from DOT Diagnostics (Prague, Czech Republic), Beckman (Fullerton, Calif., USA) and Linco Research (St. Charles, Mo., USA), respectively.

DNA isolation and genetic analysis. High molecular DNA was isolated from liver samples obtained after the rats were killed using the Genomix kit (Talent, Trieste, Italy). Microsatellite markers were chosen from the available rat linkage maps (Wellcome Trust Centre for Human Genetics, http://www. well.ox.ac.uk and the Whitehead Institute for Biomedical Research/MIT Rat Genome Map, http://www.waldo.wi.mit.edu/ rat/public). Initially, DNAs from $8 \mathrm{hHTg}$ and $2 \mathrm{BN}$ parental rats were analysed by PCR to look for polymorphism between the strains. $\mathrm{F}_{2}$ progeny were then typed for selected markers $[8,9]$. Briefly, reactions $(20 \mu \mathrm{l})$ consisted of $100 \mathrm{ng}$ DNA, $0.25 \mu \mathrm{mol} / \mathrm{l}$ of each primer, $25 \mu \mathrm{mol} / 1 \mathrm{dNTPs}, 1.5 \mathrm{mmol} / \mathrm{L}$ $\mathrm{MgCl}_{2}, 45 \mathrm{mmol} / \mathrm{L}$ Tris-HCl pH 8.8, $11 \mathrm{mmol} / \mathrm{L}(\mathrm{NH} 4)_{2} \mathrm{SO}_{4}$ $\mathrm{pH} 8.8,6.7 \mathrm{mmol} / \mathrm{l} \beta$-mercaptoethanol, $4.5 \mu \mathrm{mol} / \mathrm{l}$ EDTA and $0.4 \mathrm{U}$ Taq polymerase. The PCR program consisted of an initial 4 min at $96^{\circ} \mathrm{C}$ followed by 35 cycles of $30 \mathrm{~s}$ at $94^{\circ} \mathrm{C}, 45 \mathrm{~s}$ at $55^{\circ} \mathrm{C}$ or $60^{\circ} \mathrm{C}$, and $10 \mathrm{~s}$ at $72^{\circ} \mathrm{C}$. PCR products were resolved on either $2 \%$ agarose gels stained with ethidium bromide, or on $8 \%$ polyacrylamide gels probed with $[\alpha-32 \mathrm{P}]-$ dCTP-labelled primers.

Data analysis. Quantitative variables between the two parental strains were compared by unpaired t-test. A $p$ value of less than 0.05 was taken as significant. In the $F_{2}$ rats, normality of the quantitative traits was tested using the Anderson-Darling function on MINITAB (version 13) (Minitab, State College, Pa., USA). Due to skewness at the extremes of some of the traits, data were log transformed before further analysis. Correlation between traits in the $\mathrm{F}_{2}$ progeny was assessed by Pearson's method. Genetic linkage maps for the polymorphic markers were constructed and the location of QTLs were determined using the MAPMAKER programs $[10,11]$. LOD scores were initially computed under free assumption, but subsequently also under dominant, recessive and additive models to identify the mode of inheritance that fitted the data best. A lod score of greater than 4.3 was taken to indicate significant linkage and a lod score of greater than 2.8 suggestive linkage [12]. Co-segregation of phenotypes with alleles at marker loci was evaluated by comparing the values between different genotypes by the non-parametric Kruskal-Wallis test. A $p$ value of less than 0.0016 was taken to indicate suggestive linkage as recommended for a genome scan in an experimental inter-cross [12].

\section{Results}

The phenotypes in the parental strains and the mean values for the traits in the $\mathrm{F}_{2}$ rats are shown in Table 1. hHTg rats had a higher plasma Tg concentration and systolic and diastolic blood pressures compared with $\mathrm{BN}$ rats. However, there was no difference in fasting 


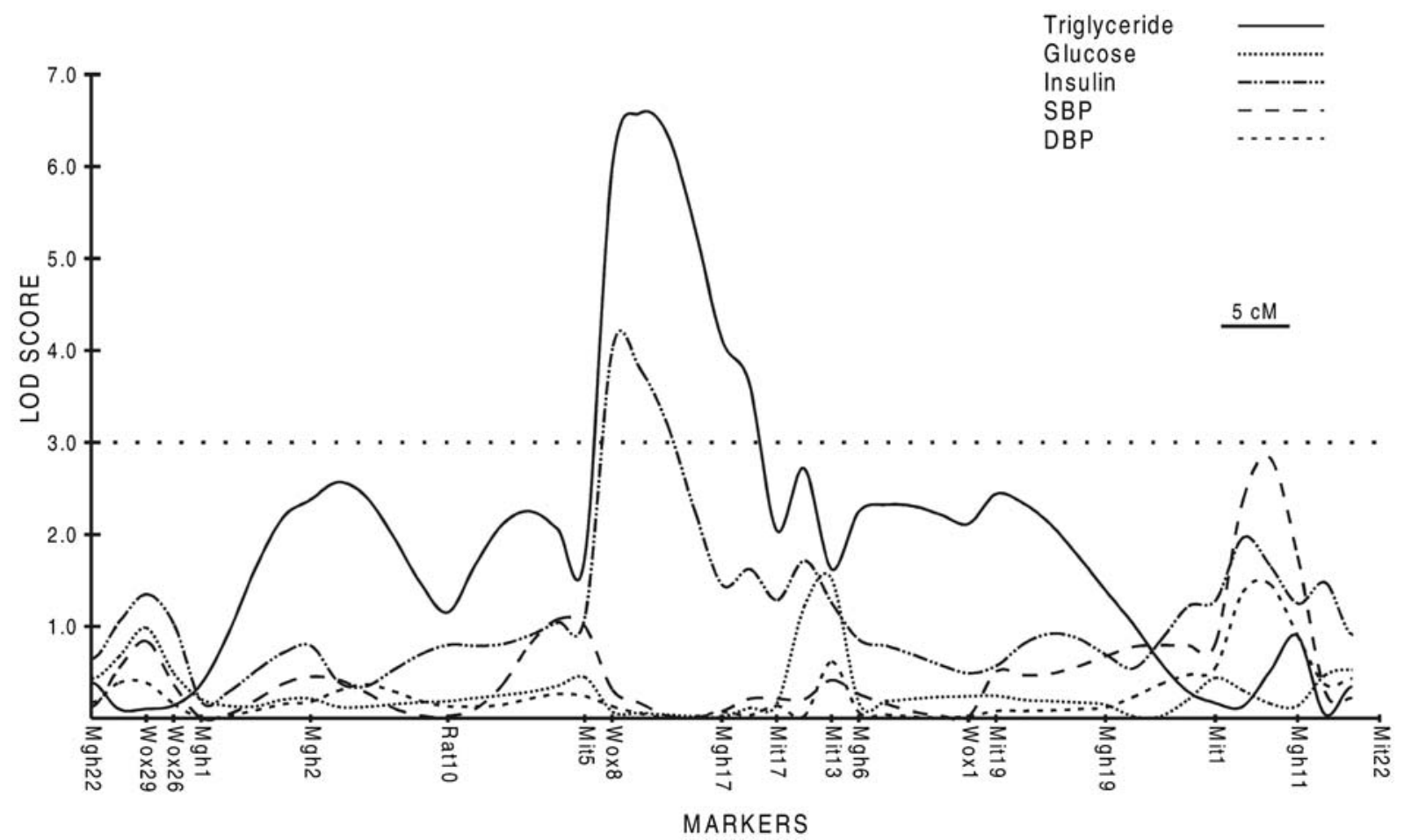

Fig. 1. A lod score plot of linkage of markers on rat chromosome 4 to fasting concentrations of plasma trigyceride, glucose and insulin and systolic (SBP) and diastolic (DBP) blood pressures measured in $\mathrm{F}_{2}$ progeny derived from an $\mathrm{hHTg} \times \mathrm{BN}$ cross. The horizontal dotted line represents a threshold lod score of 3.0. Note the lod score peaks for triglyceride and insulin concentrations

Table 1. Phenotypes in the parental and $\mathrm{F}_{2}$ rats

\begin{tabular}{lccc}
\hline Phenotype & $\mathrm{hHTg}(n=10)$ & $\mathrm{BN}(n=10)$ & $\mathrm{F}_{2}$ \\
\hline Plasma triglycerides (mmol/l) & $1.54 \pm 0.06^{*}$ & $1.24 \pm 0.04$ & $1.66 \pm 0.59(0.55-4.01(187)$ \\
Systolic BP (mm Hg) & $156.7 \pm 5.5^{*}$ & $114.9 \pm 3.4$ & $127.8 \pm 13.8(103-180)(186)$ \\
Diastolic BP (mm Hg) & $130.5 \pm 3.9^{*}$ & $95.1 \pm 3.9$ & $102.3 \pm 11.0(71-143)(186)$ \\
Plasma insulin (ng/ml) & $0.50 \pm 0.33$ & $0.54 \pm 0.24$ & $1.50 \pm 0.81(0.40-5.00)(165)$ \\
Plasma glucose (mmol/l) & $5.50 \pm 0.70$ & $5.50 \pm 0.62$ & $4.19 \pm 0.76(2.79-6.67)(172)$ \\
\hline
\end{tabular}

Means \pm SD are shown. For the F2 rats, the range is also shown, and the number of animals with available data for the phenotype. $* p<0.01$ compared with $\mathrm{BN}$ rats

insulin or glucose concentrations between the strains. In the $\mathrm{F}_{2}$ progeny, there was a positive correlation between plasma $\mathrm{Tg}$ and glucose concentrations $(r=0.222, p=0.004)$, and a negative correlation between plasma $\mathrm{Tg}$ and insulin concentrations $(r=$ $-0.198, p=0.011)$. There was no correlation between $\mathrm{Tg}$ concentration and either systolic or diastolic blood pressures or between either insulin or glucose and blood pressure values.

Altogether 464 markers were tested for polymorphism between hHTg and $\mathrm{BN}$ rats. The chromosomal distribution of the markers is shown in Table 2. Of the markers, $282(60.8 \%)$ were polymorphic. There was a low incidence (seven markers, $1.5 \%$ ) of residual heterozygosity in the hHTg rat. $\mathrm{F}_{2}$ hybrids were genotyped for $153(54.3 \%)$ of the polymorphic markers. The aim was to try and cover each chromosome with an average inter-marker distance of about 10 centimorgans (cM). However, this was not achieved for all the chromosomes (Table 2) due to the distribution of the available polymorphic markers, and some of the chromosomes were specifically enriched for additional markers based on initial results.

A QTL with an important effect on the plasma Tg concentration (peak lod score 6.5) was mapped to rat chromosome 4 in the interval between markers $D 4$ Mit5 and D4 Mit17 (Fig. 1). The model that fitted the data best was the additive (co-dominant) model and the QTL accounted for $17.9 \%$ of the variation of $\mathrm{Tg}$ concentration in the $\mathrm{F}_{2}$ population. The $95 \% \mathrm{CI}$ for the QTL (1 lod score) spanned $6 \mathrm{cM}$. The quantitative effect on plasma $\mathrm{Tg}$ concentration for alleles of the most proximate marker (D4 Wox8) are shown in Table 3. The QTL region did not have any effect on 
Table 2. Chromosomal distribution of microsatellite markers tested for polymorphism between hHTg and BN rats and genotyped in F2 hybrids

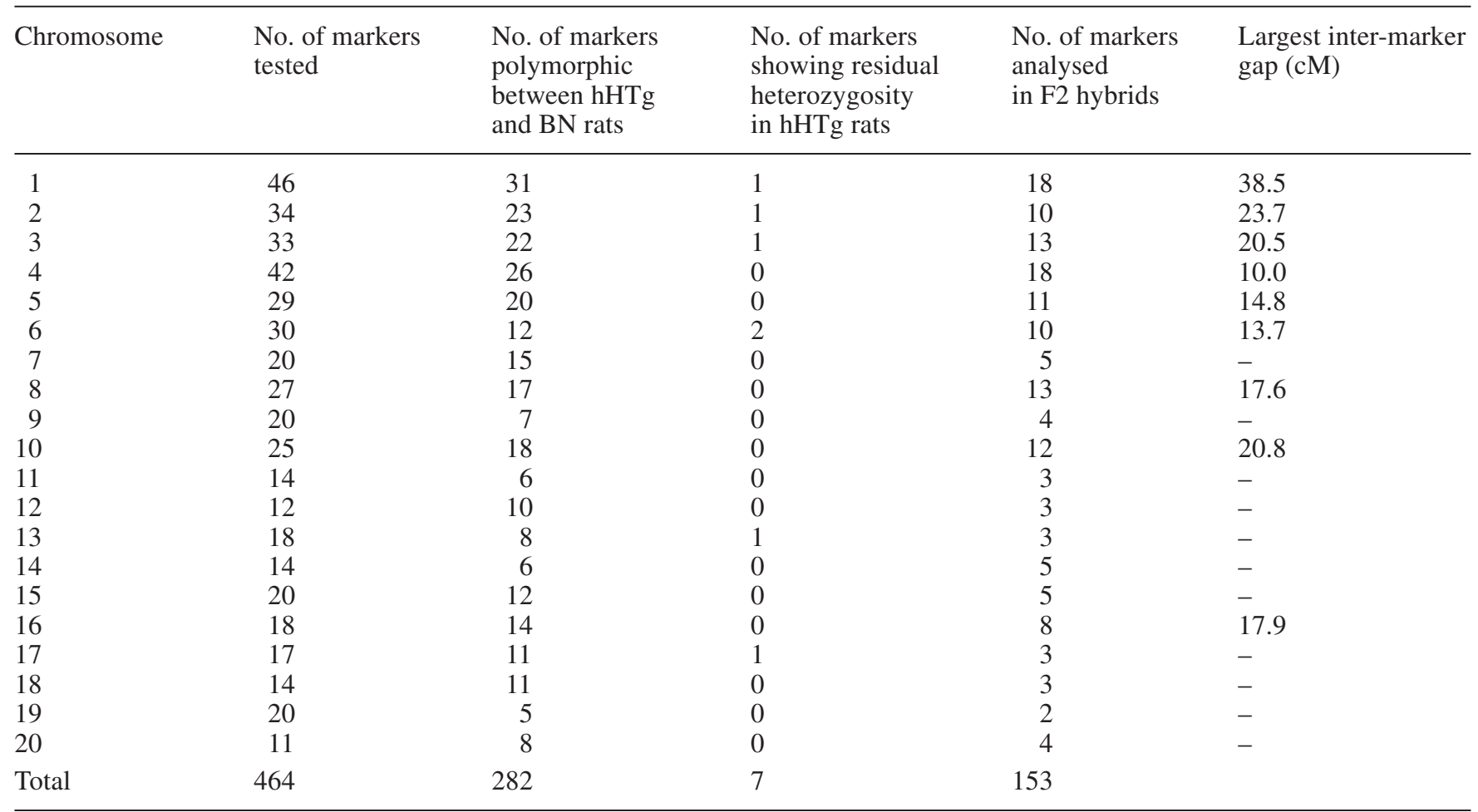

The last column shows the maximum inter-marker distance for those chromosomes where a sufficient number of markers were available to provide chromosome wide coverage

Table 3. Co-segregation of traits with genotypes at selected markers

\begin{tabular}{|c|c|c|c|c|c|}
\hline \multicolumn{6}{|l|}{ Genotype } \\
\hline Chromosome & Marker & $\mathrm{BB}$ & $\mathrm{HB}$ & $\mathrm{HH}$ & $p$ \\
\hline 4 & D4Wox8 & $1.38 \pm 0.43(43)$ & $1.63 \pm 0.55(87)$ & $1.93 \pm 0.59(42)$ & 0.0001 \\
\hline 8 & D8Mit13 & $1.39 \pm 0.38(44)$ & $1.60 \pm 0.59(87)$ & $1.87 \pm 0.60(47)$ & 0.0003 \\
\hline 8 & D8Mgh6 & $1.39 \pm 0.37(38)$ & $1.62 \pm 0.52(97)$ & $1.91 \pm 0.77(48)$ & 0.0019 \\
\hline 4 & D4Wox8 & $1.65 \pm 0.84(35)$ & $1.67 \pm 0.88(74)$ & $1.11 \pm 0.52(41)$ & 0.0007 \\
\hline 12 & D12Mit3 & $1.75 \pm 0.66(38)$ & $1.45 \pm 0.80(74)$ & $1.41 \pm 1.04(34)$ & 0.0068 \\
\hline \multicolumn{6}{|c|}{ Plasma glucose (mmol/) } \\
\hline 16 & D16Mgh3 & $4.03 \pm 0.69(40)$ & $4.15 \pm 0.80(90)$ & $4.57 \pm 0.60(38)$ & 0.0025 \\
\hline 16 & D16Mit5 & $4.05 \pm 0.69(42)$ & $4.11 \pm 0.72(81)$ & $4.53 \pm 0.60(34)$ & 0.0036 \\
\hline \multicolumn{6}{|c|}{ Diastolic blood pressure ( $\mathrm{mm} \mathrm{Hg}$ ) } \\
\hline 15 & D15Wox3 & $98.7 \pm 12.2(51)$ & $103.4 \pm 9.8(88)$ & $104.2 \pm 11.0$ & 0.0009 \\
\hline
\end{tabular}

For each trait mean \pm SD are shown. The numbers in parentheses are number of animals in the genotype group with trait data. BB, homozygous for Brown Norway allele; HB, heterozy- gote; HH, homozygote for hHTg allele. The $p$ values are from a non-parametric Kruskal-Wallis comparison of the groups 


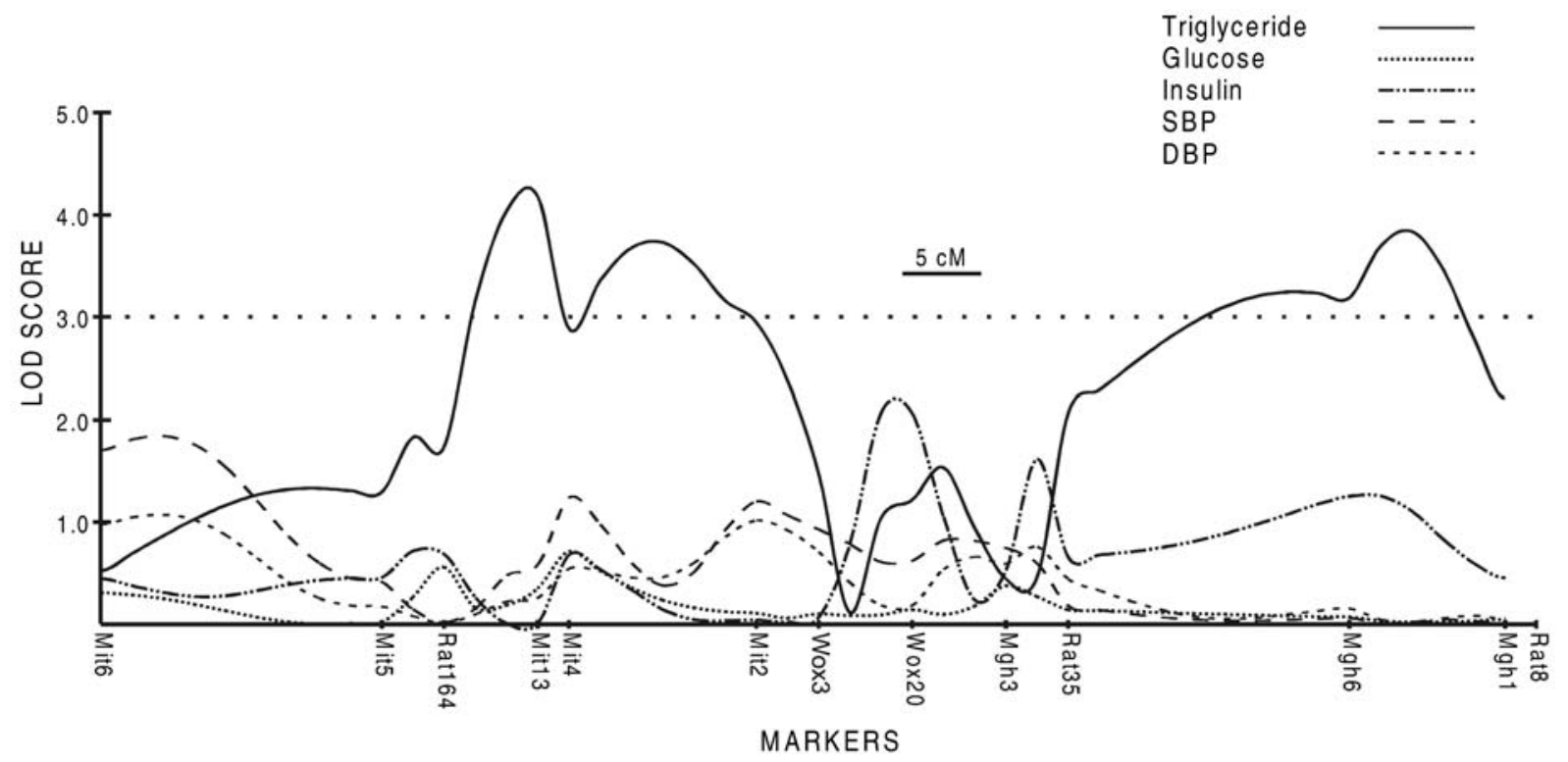

Fig. 2. A lod score plot of linkage of markers on rat chromosome 8 to fasting concentrations of plasma trigyceride, glucose and insulin and systolic (SBP) and diastolic (DBP) blood pressures measured in $\mathrm{F}_{2}$ progeny derived from an $\mathrm{hHTg} \times \mathrm{BN}$ cross. The horizontal dotted line represents a threshold lod score of 3.0. Note the multiple lod score peaks for triglyceride concentration

systolic or diastolic blood pressure or plasma glucose but did influence plasma insulin concentration (peak lod score 4.1). However, interestingly the model that best fitted the effect on insulin concentration was recessive and paradoxically the effect on insulin was opposite to what might have been expected with $\mathrm{F}_{2}$ animals carrying two copies of the hHTg allele having lower plasma insulin (Table 3).

On chromosome 8, multiple regions affecting $\mathrm{Tg}$ concentration with peaks lod scores of around 4.0 were observed in the regions between D8rat164 and D8Wox3 and again between D8Rat35 and D8Mghl (Fig. 2). The effects were additive (co-dominant) in both regions. The quantitative effects on plasma $\mathrm{Tg}$ concentration for alleles of the most proximate markers in each region are shown in Table 3. There was no evidence for QTLs affecting blood pressure or plasma insulin or glucose on chromosome 8 (Fig. 2).

None of the other chromosomes contained QTLs definitely affecting any of the traits. However, individual markers showed associations with specific traits. Specifically, two markers on chromosome 16 (D16Mgh3 and D16Mit5) co-segregated with plasma glucose concentration, while a marker on chromosome 12 (D12Mit3) related to plasma insulin concentration. A marker on rat chromosome 15 (D15Wox3) co-segregated with differences in diastolic blood pressure with $\mathrm{a} \approx 5 \mathrm{~mm} \mathrm{Hg}$ higher value in animals carrying one or two copies of the hHTg allele. Markers on chromosomes 3 and 5 had moderate effects on systolic blood pressure. For the chromosome 3 marker (D3Rat126), the hHTg allele was associated with $\mathrm{a} \approx 7 \mathrm{mmHg}$ higher blood pressure, while for chromosome 5, the hHTg allele was associated with a similar decrease in blood pressure (Table 3).

\section{Discussion}

In this study, we report that the main QTLs influencing plasma $\mathrm{Tg}$ concentration in $\mathrm{hHTg}$ rat are located on chromosomes 4 and 8 . The QTL on chromosome 4 exceeds the threshold for linkage (lod score $>4.3$ ) proposed for an experimental inter-cross, while the QTLs on chromosome 8 exceed the threshold for suggestive linkage (a lod score >2.8) [12]. Our data support and extend previous findings in other experimental models that these rat chromosomes harbour QTLs that affect plasma Tg concentration. Specifically our region on chromosome 4 overlaps with that reported by others [13] in the SHR rat and our findings indicate that if there is a single QTL affecting Tg concentration in this region of chromosome 4 , it is very likely to be localized to a $6 \mathrm{cM}$ segment between D4Wox8 and $D 4 M g h 17$. Genes with a potential impact on lipid metabolism in this region include neuropeptide-Y (Npy) and fatty acid binding protein 1 (Fabpl) [14]. However, it is important to note that the Cd36 (Fat) gene in which a mutation has been shown to cause defective fatty acid and glucose metabolism in SHR $[15,16]$ lies considerably away from the region containing our QTL. On rat chromosome 8, a QTL for plasma $\mathrm{Tg}$ at D8Mit2 in the Otsuka Long-Evans Tokushima Fatty rat has been reported [17]. This is consistent with one of the QTL regions observed in our cross . The breadth of this QTL region and the multiple peaks suggests that more than one gene affecting plasma Tg could be located here and it is notable that several apolipoprotein genes are clustered in this region of chromosome 8 [18]. Moreover, this region also over- 
laps a non-insulin dependent diabetes mellitus QTL [19]. Others have reported [20] that differential segments of chromosome 8 of BN.lx and SHR.lx congenic strains (derived from SHR and normotensive BN rats) also carry genes associated with plasma triglycerides. The pertinent chromosomal segment of the BN-lx strain includes the marker D8Mgh6 that also co-segregates with plasma $\mathrm{Tg}$ concentration in our cross.

The hypertriglyceridaemia in the hHTg rat is mainly due to an increase in circulating VLDL particles [21]. There is evidence for both increased hepatic production of VLDL [22] as well as decreased disposal of $\mathrm{Tg}$ in peripheral tissues [23]. Thus the genetic regulation of plasma $\mathrm{Tg}$ concentration in this model was expected to be complex and the finding of multiple QTLs is consistent with the known biology of the model. Mapping of the QTLs opens up the possibility of capturing individual QTLs in congenic strains [24]. Such strains will not only ultimately permit causative genes to be identified but also help to assess which aspect of Tg metabolism is affected by individual QTLs.

There is considerable uncertainty about the precise nature of the association between different features of the IR syndrome and in particular whether the correlations represent a common genetic aetiology [2, 3, 25]. The hHTg rat strain was selected solely on the basis of a plasma $\mathrm{Tg}$ response to a high monosaccharide diet [5]. Thus, the subsequent finding [4] of moderate hypertension in the model, in the context of previous data on the IR syndrome [2], strongly suggested that a common genetic basis could underlie the dyslipidaemia and hypertension. It was therefore quite surprising to observe that neither the chromosome 4 nor the chromosome $8 \mathrm{Tg}$ QTLs had any effect on BP despite the very powerful effects on $\mathrm{Tg}$ concentration. Indeed, no correlation was observed between $\mathrm{Tg}$ concentration and $\mathrm{BP}$ in the $\mathrm{F}_{2}$ rats suggesting that the association of dyslipidaemia and hypertension in the hHTg rat is simply due to genetic drift [26]. Such a conclusion is of course crucially dependent on the reliability of the BP assessment. We used a very standard method for short-term direct BP measurement in conscious unrestrained rats, similar to one that we have previously used in other hypertension co-segregation studies [27]. Furthermore, the same technique easily showed a difference in SBP and DBP between the two progenitor strains and in the $F_{2}$ rats the full range of readings between hHTg and BN rats was observed. Finally, although our genome scan is not complete, data on individual markers suggest that the quality of the data is sufficient to detect modest differences in BP between genotype groups. Thus, it seems unlikely that an effect on BP was missed on chromosomes 4 and 8 or indeed on other well-covered chromosomes such as 1,2 and 10 , where BP QTLs have been located in other experimental crosses [28].

Although there were no differences in either plasma insulin or glucose concentrations between $\mathrm{hHTg}$ and $\mathrm{BN}$ rats, both phenotypes showed a considerable range of values in $\mathrm{F}_{2}$ rats and co-segregation with some markers. This is not an unusual occurrence for phenotypes that are under polygenic control some of which are likely to differ between the two progenitor strains [26]. A suggestive QTL was detected on chromosome 4, coincident with the Tg QTL, that influenced fasting plasma insulin concentration. Given the strong evidence that the hHTg rat shows resistance to the effect on insulin in peripheral tissues $[4,6,22]$ it was surprising to see that the quantitative effect on insulin went in the opposite direction to that on $\mathrm{Tg}$. Thus, $\mathrm{F}_{2}$ rats homozygote for the hHTg $(\mathrm{HH})$ allele animals had an approximately 50\% lower fasting plasma insulin as compared to $\mathrm{BB}$ or $\mathrm{BH}$ rats It is conceivable that the co-localisation of the QTLs for insulin and $\mathrm{Tg}$ is incidental and that they represent the effect of different genes, especially as the mode of inheritance seems to be different. However, another plausible explanation is that the lower insulin concentration in these animals is secondary to a toxic effect of $\mathrm{Tg}$ on insulin secretion by the islets of Langerhans. Accumulation of $\mathrm{Tg}$ in the pancreas particularly in the context of the IR syndrome has been shown to impair glucose-stimulated insulin secretion by beta cells [29, 30].

In summary, we have mapped the major QTLs that influence plasma Tg concentration in the hHTg rat and have shown that the same QTLs do not influence BP in this model of the IR syndrome. Identification of the genes that influence $\mathrm{Tg}$ concentration through further analysis of the mapped regions should improve our understanding of the regulation of an important risk factor for cardiovascular disease. Our findings call for a further appraisal of the precise nature of the relationships between different components of the IR syndrome.

Acknowledgements. This work was supported by research grants of the Slovak Agency for Science (GAV No. 544/ 1993-1996, No. 4131/1997-2000), by a research grant of the Slovak Grant Agency for Technic (GAT PECO 931004) within the frame of the EURHYPGEN 1 and 2 Concerted Action of the EU. Genotype analysis was supported by a grant from the British Heart Foundation (PG97026). We greatly appreciate the expert assistance of Dana Jurkovicova, $\mathrm{PhD}$ and the technical assistance of Ms. A. Mitkova, and S. Kuklova.

\section{References}

1. Reaven GM (1988) Role of insulin resistance in human disease. Diabetes 37:1595-1607

2. Groop L, Orho-Melander M (2001) The dysmetabolic syndrome. J Intern Med 250:105-120

3. Poulsen P, Vaag A, Kyvik K, Beck-Nielsen H (2001) Genetic versus environmental aetiology of the metabolic syndrome among male and female twins. Diabetologia 44:537-543

4. Klimes I, Sebökova E, Vrana A et al. (1995) The hereditary hypertriglyceridemic rat, a new animal model of the insulin resistance syndrome. In: Shafrir E (ed.) Lessons from animal diabetes V. Smith-Gordon, pp 271-283 
5. Vrana A, Kazdova L (1990) The hereditary hypertriglyceridemic non-obese rat: an experimental model of human hypertriglyceridemia. Transplant Proc 22:2579

6. Stolba P, Opltova H, Husek P et al. (1993) Adrenergic overactivity and insulin resistance in nonobese hereditary hypertriglyceridemic rats. Ann NY Acad Sci 683:281-288

7. Vrana A, Kazdova L, Dobesova Z et al. (1993) Triglyceridemia, glucoregulation and blood pressure in various rat strains. Effects of dietary carbohydrates. Ann NY Acad Sci 683:57-68

8. Dubay C, Vincent M, Samani NJ et al. (1993) Genetic determinants of diastolic and pulse pressure map to different loci in Lyon hypertensive rats. Nat Genet 3:354-357

9. Samani NJ, Gauguier D, Vincent M et al. (1996) Analysis of quantitative trait loci for blood pressure on rat chromosomes 2 and 13: age-related differences in effect. Hypertension 28:1118-1122

10. Lander ES, Green P, Abrahamson J et al. (1987) MAPMAKER: An interactive computer package for constructing primary genetic linkage maps of experimental and natural populations. Genomics 1:174-181

11. Lander E, Botstein D (1989) Mapping mendelian factors underlying quantitative traits using RFLP linkage maps. Genetics 12:185-199

12. Lander E, Kruglyak L (1995). Genetic dissection of complex traits: guidelines for interpreting and reporting linkage studies. Nat Genet 11:241-247

13. Kovacs P, Klöting I (1998) Quantitative trait loci on chromosomes 1 and 4 affect lipid phenotypes in the rat. Arch Biochem Biophys 354:1-5

14. Bihoreau M-T, Sebag-Montefiore L, Godfrey RF et al. (2001) A high resolution consensus linkage map of the rat, integrating radiation hybrid and genetic maps. Genomics 75:57-69

15. Aitman TJ, Glazier AM, Wallace CA et al. (1999) Identification of $\mathrm{Cd} 36$ (Fat) as an insulin resistance gene causing defective fatty acid and glucose metabolism in hypertensive rats. Nat Genet 21:76-83

16. Pravenec M, Landa V, Zidek V et al. (2001) Transgenic rescue of defective $\mathrm{Cd} 36$ ameliorates insulin resistance in spontaneously hypertensive rats. Nat Genet 27:156-158

17. Okuno S, Watanabe TK, Ono T et al. (1999) Genetic determinants of plasma triglyceride levels in (OLETF x BN) $\mathrm{x}$ OLETF backcross rats. Genomics 62:350-355
18. Koike G, Miano JM, Vanvooren P, Shiozawa M, Szpirer C, Jacob HJ (1998) Mapping of the ratSM22 gene to chromosome 8q24: a candidate for high blood pressure and cardiac hypertrophy. Mamm Genome 9:76-77

19. Wei S, Wei K, Moralejo DH et al. (1999) Mapping and characterization of QTL for non-insulin-dependent diabetes mellitus with an improved genetic map in the Otsuka LongEvans Tokushima Fatty rat. Mamm Genome 10:249-258

20. Kren V, Krenova D, Pravenec M, Zdobinska M (1995) Chromosome 8 congenic strains: tools for genetic analysis of limb malformation, plasma triglycerides, and blood pressure in the rat. Folia Biol (Praha) 41:284-293.

21. Sebokova E, Klimes I, Hermann M et al. (1992) Fish oil modifies lipid composition and liver LDL receptor activity in hypertriglyceridemic rats. Diabetes Nutr Metab 5:249-257

22. Sebokova E, Klimes I, Knopp J et al. (1996) Regulation of gene expression for lipogenic enzymes in the liver and adipose tissue of hereditary hypertriglyceridemic, insulin resistant rats: effect of dietary sucrose and marine fish oil. Biochim Biophys Acta1303:56-62

23. Ukropec J, Klimes I, Gasperikova D et al. (2002) An Increase in Peroxisomal Fatty Acid Oxidation Is Not Sufficient to Prevent Tissue Lipid Accumulation in hHTg Rats. Ann NY Acad Sci 967:71-79

24. Rapp JP, Deng AY (1995) Detection and positional cloning of blood pressure quantitative trait loci: is it possible? Hypertension 25:1121-1128

25. Liese AD, Mayer-Davies EJ, Tyroler HA et al. (1997) Familial components of the multiple metabolic syndrome: the ARIC study. Diabetologia 40:963-970

26. Rapp JP (1983) Genetics of experimental and human hypertension. In: Genest J, Kuchel O, Hamet P, Cantin M (eds) Hypertension. McGraw-Hill, New York, pp 582-598

27. Samani NJ, Lodwick D, Vincent M et al. (1993) A gene differentially expressed in the kidney of the spontaneously hypertensive rat cosegregates with increased blood pressure. J Clin Invest 92:1099-1103

28. Rapp JP (2000) Genetic analysis of inherited hypertension in the rat. Physiol Rev 80:135-171

29. McGarry JD, Dobbins RL (1999) Fatty acids, lipotoxicity and insulin secretion. Diabetologia 42:128-138

30. McGarry JD (2002) Banting lecture 2001: Dysregulation of fatty acid metabolism in the etiology of type 2 diabetes. Diabetes 51:7-18 